“C 2018 IEEE. Personal use of this material is permitted. Permission from IEEE must be obtained for all other uses, in any current or future media, including reprinting/republishing this material for advertising or promotional purposes, creating new collective works, for resale or redistribution to servers or lists, or reuse of any copyrighted component of this work in other works." 


\title{
Design, Manufacturing and Optimization of PM-SMC Motors
}

\author{
Bo Ma, Gang Lei, and Jianguo Zhu \\ School of Electrical and Data Engineering, University of Technology Sydney, \\ NSW 2007, Australia \\ Bo.Ma@student.uts.edu.au
}

\begin{abstract}
Soft magnetic composite (SMC), as a relatively new magnetic material, has attracted much attention in the design of electrical machines. This paper aims to discuss the specific and common problems in the design of permanent magnet (PM) motors with SMC cores. Firstly, the motor topology design is introduced for taking advantage of the SMC properties. Then, precise material testing and modeling methods are presented for the accurate core loss calculation of PM-SMC motors. Considering the influence of manufacturing process, two design optimization approaches are utilized for enhancing the motor performance. For improving the electromagnetic properties of the SMC cores, the orthogonal experiment is proposed for investigating and optimizing the heat treatment process. Considering the manufacturing tolerances in the actual production, the robust tolerance design optimization approach is introduced for pursuing the optimal motor performance and manufacturing cost with high reliability.
\end{abstract}

Keywords - Soft magnetic composite, core loss; manufacturing tolerances; orthogonal experiment design; robust optimization.

\section{INTRODUCTION}

SMC material has been widely investigated for the development of PM motors in recent years [1]-[4]. The basic SMC particles are of $0.05-0.1 \mathrm{~mm}$ in diameter coated with very thin electrical insulation as illustrated in Fig. 1(a) [1]. Compared with the traditional silicon steel sheet, special properties of SMC cores can be synthesized in a few points. Firstly, SMC has the isotropic performance in electromagnetic and thermal properties because of the powder nature, which can be applied to electromagnetic devices design with three-dimensional flux path. The eddy current loss is very low due to the isolation coat of the particles, which also leads to much lower magnetic permeability compared with silicon steel. Secondly, the structure can be pressed by a mold, which offers an easier way for motor manufacturing. However, this manufacturing method brings out much higher hysteresis loss and weaker mechanical strength.

Fig. 1 (b) illustrates the manufacturing process of an SMC component, which contains material production, compaction and heat treatment, etc. Compared with the traditional electrical machine core production with laminated silicon steels, this process influences the electrical machine performance from different aspects, including the compaction density and heat treatment [1].
This paper presents a review of design, analysis and optimization approaches of the PM electrical machine with SMC. Firstly, the PM motor design features with SMC is introduced. As one of the difficulties of accurately calculating the performance of SMC motors, the core loss testing and modeling methods are then discussed. Considering the manufacturing influence, an orthogonal design of experiment is then presented for investigating the factors existing in the heat treatment process and optimizing the procedure. Finally, considering the uncertainties in the actual manufacturing (e.g., manufacturing tolerances), the robust optimization technique named design for six-sigma (DFSS) and the DFSS based robust tolerance optimization approach is presented to improve the performance and manufacturing quality of PM-SMC motors with a design example.

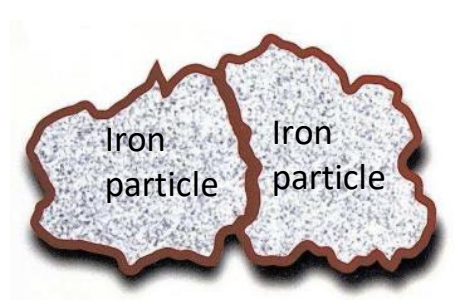

(a)

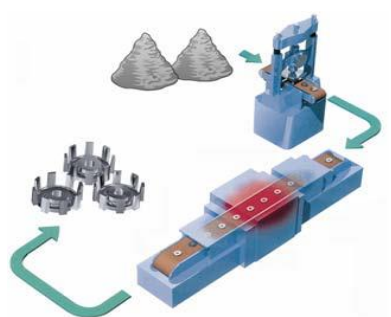

(b)
Fig. 1. (a) SMC powder with insulation, (b) SMC core production process

\section{DESIGN OF SMC MOTORS}

Due to the isotropic magnetic properties and flexible manufacturing properties, SMC material benefits the electrical machine design with three-dimensional flux paths, such as claw pole motors and transverse flux motors [4]-[6]. Compared with the 3D flux motor design with laminated steel sheets, the situation that flux crosses the laminated steel can be avoided. To present a specific example, Fig. 2 shows the topology of a new 3D-flux flux-switching permanent magnet machine. SMC cores are applied to both the rotor and stator parts. The flux passes through the PM in the circumferential direction, teeth in radial direction and rotor in the axial direction.

The coated particle component of SMC leads to the different electromagnetic properties compared with silicon steel sheets, which include low relative permeability, high hysteresis loss, etc. Several design techniques or rules can be used to compensate or avoid the drawbacks effectively. Since the hysteresis dominates the core loss, while eddy current is much lower than electrical steel, the core loss is comparable with the electrical steel at 
several hundreds of hertzes. Therefore, the high excitation frequency is usually selected at the rated operation point. On the other hand, permanent magnets are usually used for excitation to compensate the low relative permeability. Moreover, global winding as shown in Fig. 2 and flux concentrating structure also benefit the SMC motor design.

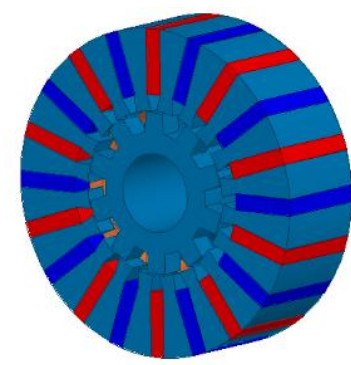

(a)

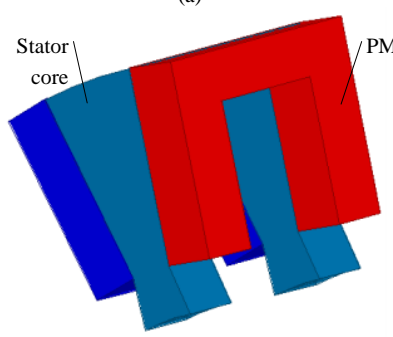

(c)

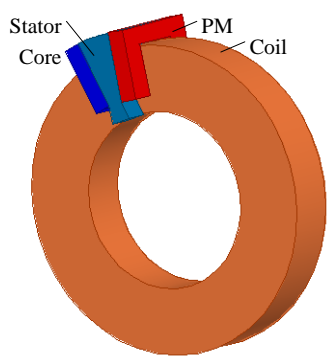

(b)

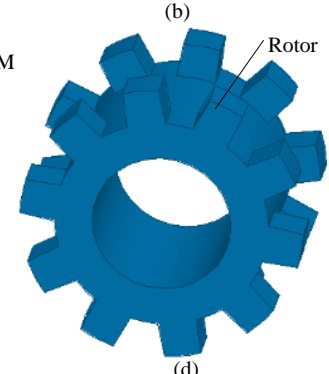

Fig. 2. Topology of a flux-switching permanent magnet machine with SMC cores

\section{CORE LOSS MEASUREMENT AND MODELING}

\section{A. Magnetic Property Testing}

To achieve the precise magnetic property model of the SMC material samples including the B-H curves, alternating loss and rotational loss, a 3-D magnetic property testing system developed by the authors' research group was taken advantage. The SMC sample was measured under 1-D alternating field excitation along three axes, and under 2-D circularly rotating field excitations at a series of excitation frequencies [7]. According to the measured - relations, the total core loss in the SMC sample can be calculated according to the Poynting's Theorem as

$$
\begin{aligned}
P_{t} & =\frac{1}{T \rho} \int_{0}^{H} \boldsymbol{H} \cdot \frac{d \boldsymbol{B}}{d t} d t \\
& =\frac{1}{T \rho} \int_{0}^{T}\left(H_{x} \frac{d B_{x}}{d t}+H_{y} \frac{d B_{y}}{d t}+H_{z} \frac{d B_{z}}{d t}\right) d t
\end{aligned}
$$

where $T$ is the period of magnetization and $\rho$ is the mass density of the sample. Fig. 3 shows the testing results of a sample of the alternating and rotational core losses.

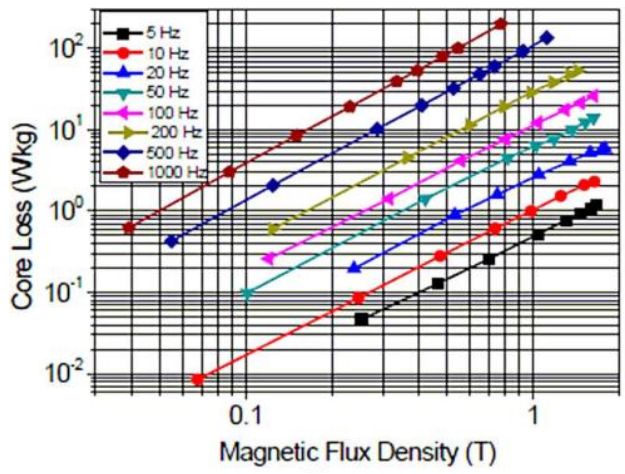

(a)

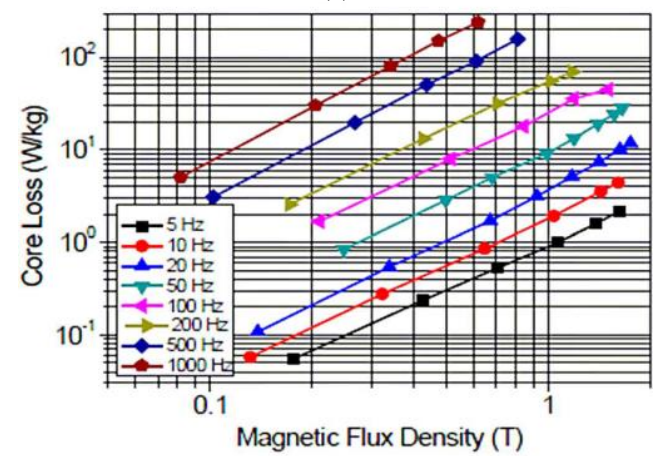

(b)

Fig. 3. (a) Alternating core losses, (b) rotational core losses

\section{B. SMC Core Loss Modeling}

Since SMC motors usually have high core loss because of the hysteresis loss performance and high excitation frequency. To calculate the electromagnetic performance precisely, the alternating and rotational core losses tested above should be modeled for motor performance calculation [8].

The alternating core loss $P_{a}$ is computed by

$$
P_{a}=C_{h a} f B_{P}{ }^{h}+C_{e a}\left(f B_{P}\right)^{2}+C_{a a}\left(f B_{P}\right)^{1.5}
$$

where $f$ is the excitation frequency, $B_{P}$ is the magnitude of the magnetic flux density, and $C_{h a}, C_{e a}, C_{a a}, h$ are all of the alternating core loss coefficients.

When the material is under two-dimensional circularly rotating flux excitation, the rotational core loss is expressed by

$$
P_{r}=P_{h r}+C_{e r}\left(f B_{P}\right)^{2}+C_{a r}\left(f B_{P}\right)^{1.5}
$$

where $P_{h r}$ is the hysteresis loss, $B_{P}$ is the magnitude of the circular flux density; $C_{e r}$ and $C_{a r}$ are rotational core loss coefficients. With an elliptical rotating flux, the core loss can be calculated by

$$
P_{e r}=R_{B} P_{r}+\left(1-R_{B}\right)^{2} P_{a}
$$

where $R_{B}=B_{m i n} / B_{m a j}, B_{\min }$ and $B_{m a j}$ are the values of the major and minor axes of the ellipse, respectively, $P_{a}$ is the alternating core loss, and $P_{r}$ is the rotational core loss under two-dimensional circularly rotating flux excitation. 


\section{MANUFACTURING AND ROBUST TOLERANCE OPTIMIZATION}

\section{A. Factor Investigation of SMC Core Properties}

For actual production, special considerations should be given to the SMC core manufacturing as the molding techniques are required. Heat treatment is also required for enhancing the magnetic and mechanical properties. Fig. 4 illustrates the manufactured core samples of 3D-flux flux-switching permanent magnet machine after the hydraulic compaction and heat treatment. (a)

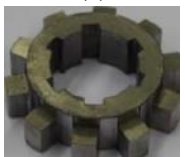

(e)

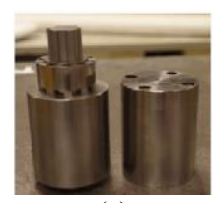

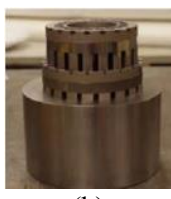

(b)

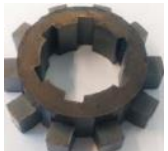

(f)

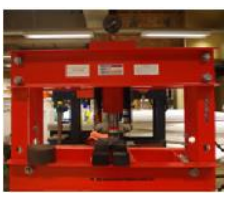

(c)

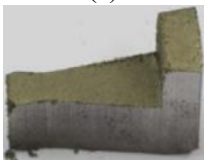

(g)

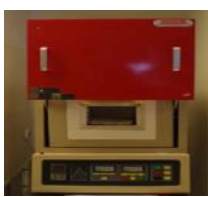

(d)

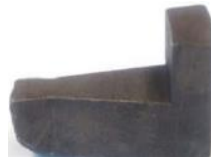

(h)
Fig. 4. Die tools, facilities and SMC cores for the proposed 3D-flux fluxswitching permanent magnet machine, (a) die tools for compaction of rotor core, (b) die tools for compaction of stator cores, (c) compact machine, (d) furnace, (e) rotor core before heat treatment, (f) rotor core after heat treatment, (g) stator core before heat treatment and $(\mathrm{h})$ stator core after heat treatment

\section{TABLE I}

EXPERIMENT DESIGN FACTORS AND LEVELS

\begin{tabular}{cccccc}
\hline \hline Factors & $\begin{array}{c}1 . \mathrm{Te} 1 \\
\left({ }^{\circ} \mathrm{C}\right)\end{array}$ & $\begin{array}{c}2 . \mathrm{Te} 2 \\
\left({ }^{\circ} \mathrm{C}\right)\end{array}$ & $\begin{array}{c}3 . \mathrm{Ti} 1 \\
(\mathrm{~min} .)\end{array}$ & $\begin{array}{c}4 . \mathrm{Te} 3 \\
\left({ }^{\circ} \mathrm{C}\right)\end{array}$ & $\begin{array}{c}5 . \mathrm{Ti} 2 \\
(\mathrm{~min} .)\end{array}$ \\
\hline Level 1 & 25 & 420 & 30 & 500 & 10 \\
Level 2 & 100 & 450 & 60 & 550 & 30 \\
Level 3 & 200 & 480 & 90 & 600 & 50 \\
\hline \hline
\end{tabular}

In the previous research, both of the compaction density and heat treatment process influence the electromagnetic properties of the SMC core [1]. Fig. 5 illustrates the influence of core density on the B-H curve [2]. In this section, the application of orthogonal experiment design is presented for investigating the effect of heat treatment process on the core performances in more detail. Fig. 6 shows the proposed heat treatment process of the compact SMC core, where Te1 is the initial temperature. The basic effect of temperature $\mathrm{Te} 2$ is to ensure the mechanical strength of the compacted SMC core. The effect of temperature Te3 is to eliminate the stress and improve the magnetic performance. To find the best heat treatment process efficiently, a 5-factor-3-level orthogonal experiment was designed, which contains only 18 samples. The factors and their levels are listed in Table I and the 18 samples are listed in Table II.

Fig.7 illustrates the hysteresis loop of two samples under $50 \mathrm{~Hz}$ alternating excitation, which indicates the hysteresis loss varies from each other. To calculate the influence of each factor on the alternating core loss, the mean and variance values can be computed by

$$
M c=\frac{1}{n} \cdot \sum_{i=1}^{n} c l(\mathrm{i})
$$

$$
\begin{aligned}
& M c l=\frac{1}{n_{L}} \cdot \sum_{i=1}^{n_{L}} c l(\mathrm{i}) \\
& V c l=\frac{1}{m_{L}} \cdot \sum_{j=1}^{m_{L}}(\operatorname{Mcl}(j)-M c)
\end{aligned}
$$

where $M c$ is the mean core loss values of the 18 samples, $n$ is the sample amount (18), and $\mathrm{cl}$ is the core loss of each sample. $\mathrm{Mcl}$ is the mean core loss of each level, $n_{L}$ is the sample amount of each Level. $V c l$ is the variance of each factor, and $m_{L}$ is the level amount. The mean core loss of each level is shown in Fig. 8. Fig. 9 illustrates the variance value and verifies that the factor 4 influence the core loss most among the five factors.

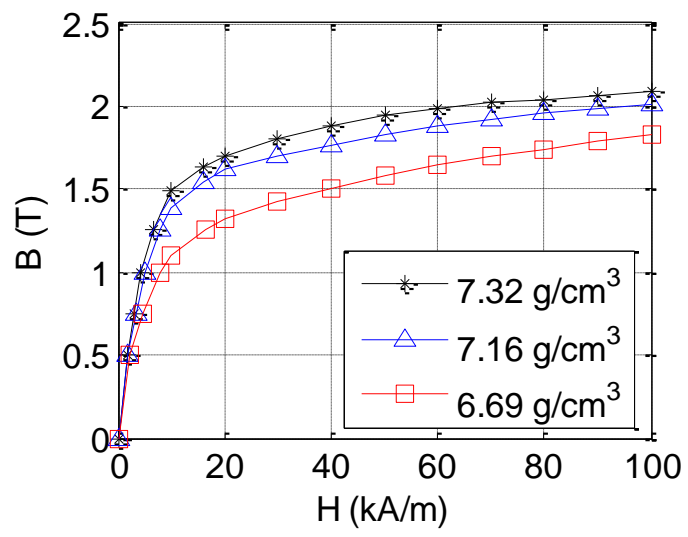

Fig. 5. B-H curves for cores of different densities

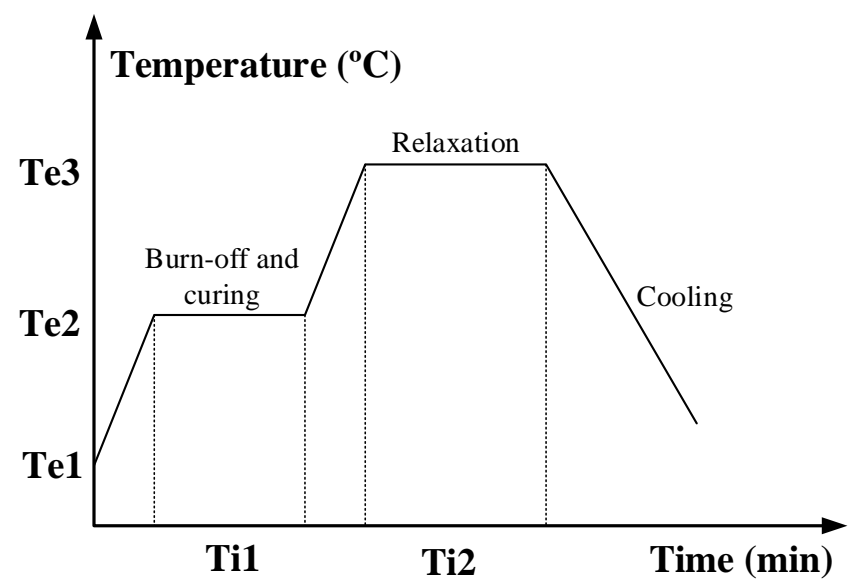

Fig. 6. Heat treatment process of the compact SMC core

As mentioned above, the same analysis can be conducted for calculating the influence of different factors on relative permeability. With the first round experiment, sample 18 is verified as the best choice of the combination. To achieve the optimized design of the heat treatment process, a new cycle of the orthogonal experiment can be designed according to the last optimal combination. Even though the optimal heat treatment process may vary because of the different SMC components' weight and dimension, the orthogonal experiment design can be an effective approach for obtaining the best procedure. 


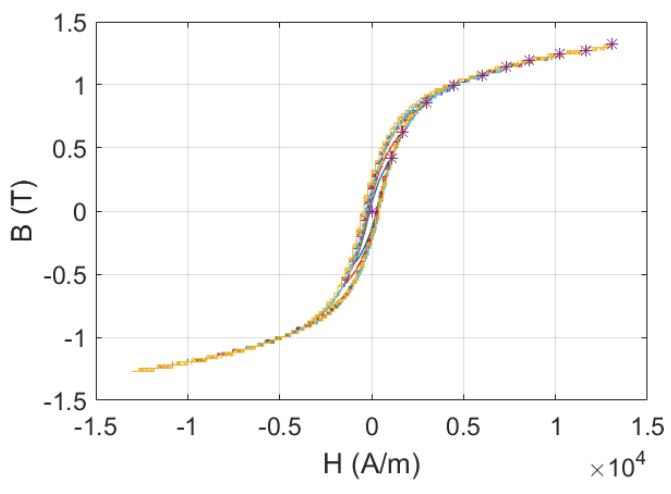

(a)

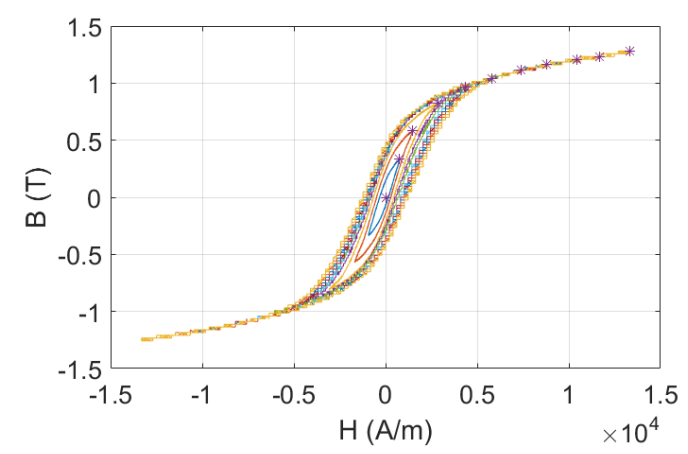

(b)

Fig. 7. Magnetic hysteresis loops of the rotor core with $50 \mathrm{~Hz}$ alternating excitation (a) sample 7, (b) sample 8

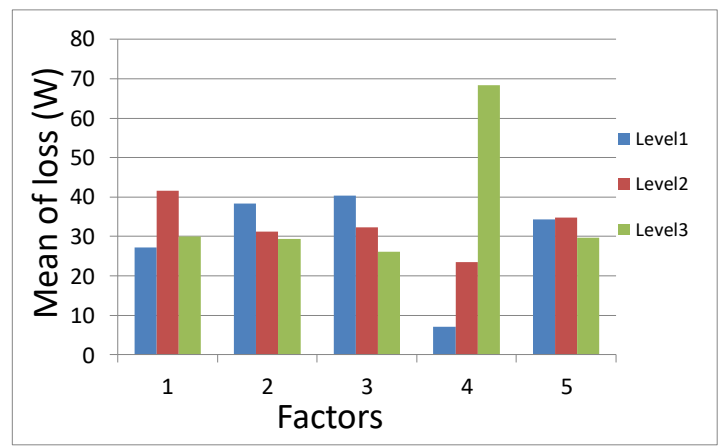

Fig. 8. Mean of alternating core loss value of each level about the five factors

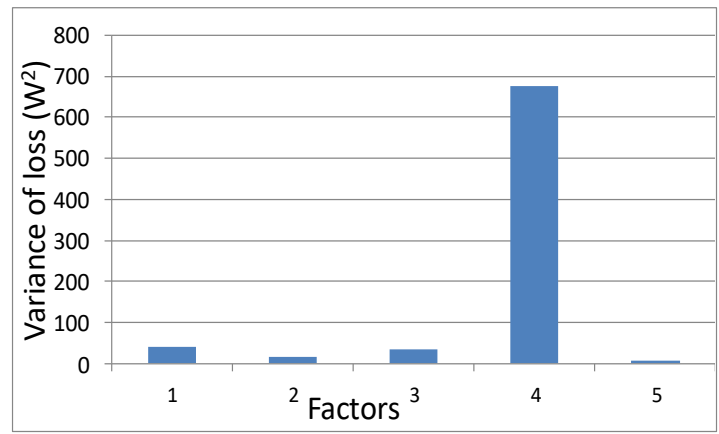

Fig. 9. Variance analysis of each factor

TABLE II

ORTHOGONAL EXPERIMENT DESIGN

\begin{tabular}{cccccc}
\hline $\begin{array}{c}\text { Sample } \\
\text { Num. }\end{array}$ & $\begin{array}{c}1 . \mathrm{Te} 1 \\
\left({ }^{\circ} \mathrm{C}\right)\end{array}$ & $\begin{array}{c}2 . \mathrm{Te} 2 \\
\left({ }^{\circ} \mathrm{C}\right)\end{array}$ & $\begin{array}{c}3 . \mathrm{Ti} 1 \\
(\mathrm{~min} .)\end{array}$ & $\begin{array}{c}4 . \mathrm{Te} 3 \\
\left({ }^{\circ} \mathrm{C}\right)\end{array}$ & $\begin{array}{c}5 . \mathrm{Ti} 2 \\
(\mathrm{~min} .)\end{array}$ \\
\hline 1. & 25 & 420 & 30 & 500 & 10 \\
2. & 25 & 450 & 60 & 550 & 30 \\
3. & 25 & 480 & 90 & 600 & 50 \\
4. & 100 & 420 & 30 & 550 & 30 \\
5. & 100 & 450 & 60 & 600 & 50 \\
6. & 100 & 480 & 90 & 500 & 10 \\
7. & 200 & 420 & 60 & 500 & 50 \\
8. & 200 & 450 & 90 & 550 & 10 \\
9. & 200 & 480 & 30 & 600 & 30 \\
10. & 25 & 420 & 90 & 600 & 30 \\
11. & 25 & 450 & 30 & 500 & 50 \\
12. & 25 & 480 & 60 & 550 & 10 \\
13. & 100 & 420 & 60 & 600 & 10 \\
14. & 100 & 450 & 90 & 500 & 30 \\
15. & 100 & 480 & 30 & 550 & 50 \\
16. & 200 & 420 & 90 & 550 & 50 \\
17. & 200 & 450 & 30 & 600 & 10 \\
18. & 200 & 480 & 60 & 500 & 30 \\
\hline \hline
\end{tabular}

\section{B. Robust Design Optimization}

As mentioned above, we investigated the influence of the specific manufacturing process on the performance of the SMC core. Commonly, when we take the manufacturing process into account, the impact of the uncertainty existing in actual manufacturing cannot be negligible [2]. Table III lists manufacturing and material variations in PM motors. To include the uncertainty information in the motor design optimization, the DFSS technique can be applied. In the DFSS, all design parameters (including material and dimension) are assumed to follow normal distributions with different means and standard deviations, thus to reflect the variations caused by material diversity and manufacturing tolerances. By adopting six sigma quality control, robust design with high quality and low failure rate can be reached [2] [9]-[11].

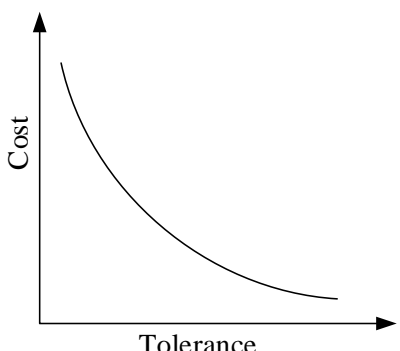

Fig. 10. Relationship between manufacturing cost and tolerance

Furthermore, the manufacturing tolerances are usually subject to manufacturing techniques and cost, which is adjustable in actual production. The relationship between manufacturing cost and tolerance is illustrated in Fig.10. As known, when we consider tolerances in the optimization process, wider tolerance usually means low manufacturing cost, but high deviation of performance. Optimizing the tolerance with the design parameters offers more design freedom on balancing performance, reliability, and cost. Therefore, 
conducting the robust tolerance optimization may integrate the robust design optimization with manufacturing in more depth. For electrical machine design, it is a multiphysics problem, more sensitive to the tolerances, and high reliability is required. Therefore, more comprehensive and effective performance analysis methods with DFSS should be developed for robust tolerance optimization of electrical machines.

TABLE III

MANUFACTURING AND MATERIAL VARIATIONS IN PM MOTORS

\begin{tabular}{ccc}
\hline \hline Description & Ideal & Variation \\
\hline Magnet dimension & Nominal & Nominal $\pm \Delta$ Tol \\
Magnet strength & Nominal & Nominal $\pm 5 \%$ \\
Magnet disposition & $0 \mathrm{deg}$ & $1.0 \mathrm{deg}$ \\
Magnetization offset & $0 \mathrm{deg}$ & $1.0 \mathrm{deg}$ \\
Skew error & Nominal & Nominal $\pm 0.67 \mathrm{deg}$ \\
Copper diameter & Nominal & Nominal $\pm \Delta \mathrm{Tol}$ \\
Eccentricity & $0 \mathrm{~mm}$ & $0.35 \mathrm{~mm}$ \\
\hline \hline
\end{tabular}

$\Delta$ Tol stands for manufacturing tolerance

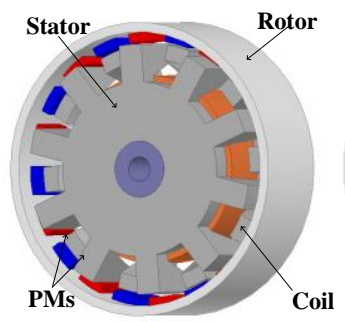

(a)

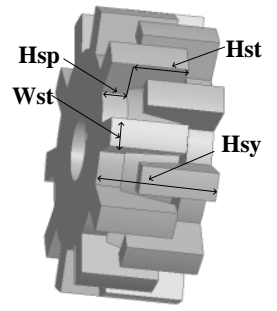

(c)

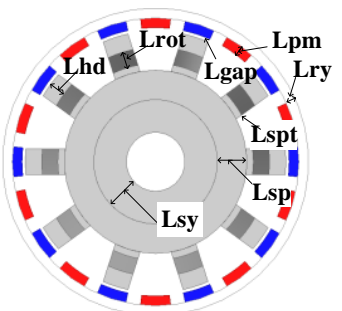

(b)

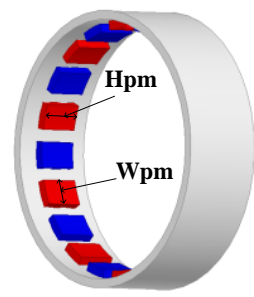

(d)
Fig. 11. Main 3D structure parameters (a) main structure of one stack, (b) parameters in radial direction,(c) parameters of stator in circumferential and axial directions, (d) parameters of PM

In this part, a design example of a claw pole motor with SMC is presented to show the effectiveness of the robust tolerance optimization with DFSS [12]. Fig. 11 shows the main stator structure. It was designed to deliver an output power of $500 \mathrm{~W}$ at $1800 \mathrm{r} / \mathrm{min}$. As listed in Table IV, 13 parameters are selected by sensitivity analysis for conducting the optimization. In this case, the optimization model is defined as

$$
\begin{aligned}
& \min : f\left(\boldsymbol{x}, \boldsymbol{\delta}_{\boldsymbol{x}}\right)=\text { mean }\left(\frac{P_{\text {initial }}}{P}+\frac{\eta_{\text {initial }}}{\eta}\right) \\
& \text { s.t. }\left\{\begin{array}{l}
g_{1}\left(\boldsymbol{x}, \boldsymbol{\delta}_{\boldsymbol{x}}\right)=500-P \leq 0, g_{2}\left(\boldsymbol{x}, \boldsymbol{\delta}_{\boldsymbol{x}}\right)=0.815-\eta \leq 0, \\
g_{3}\left(\boldsymbol{x}, \boldsymbol{\delta}_{\boldsymbol{x}}\right)=s f-0.7 \leq 0, g_{4}\left(\boldsymbol{x}, \boldsymbol{\delta}_{x}\right)=T_{\text {coil }}-75 \leq 0, \\
g_{5}\left(\boldsymbol{x}, \boldsymbol{\delta}_{\boldsymbol{x}}\right)=\text { Cost }- \text { Cost }_{\text {initial }} \leq 0, \\
g_{6}\left(\boldsymbol{x}, \boldsymbol{\delta}_{\boldsymbol{x}}\right)=\text { std }(P)-1.5 \leq 0
\end{array}\right.
\end{aligned}
$$

where $\boldsymbol{\delta}_{\boldsymbol{x}}$ means the tolerances of the design parameters, the parameters of with the initial subscript mean the initial performance values. $P$ is the output power, $T_{\text {coil }}$ is the temperature rise of the coil, $s f$ is the slot fill rate, and $\eta$ is the efficiency. Considering the reduction of the standard deviation (std) of the $P$, its $s t d$ value is controlled less than 1.5W [12].

TABLE IV

INITIAL DESIGN OF THE CPM

\begin{tabular}{ccccc}
\hline \hline Par. & Description & Unit & Initial & Tol \\
\hline$L_{s y}$ & Length of stator yoke & $\mathrm{mm}$ & 10 & 0.06 \\
$L_{s p}$ & Length of stator plate & $\mathrm{mm}$ & 10 & 0.06 \\
$L_{r o t}$ & Length of tooth root & $\mathrm{mm}$ & 5 & 0.06 \\
$L_{h d}$ & Length of tooth head & $\mathrm{mm}$ & 5 & 0.06 \\
$L_{g a p}$ & Length of air gap & $\mathrm{mm}$ & 1 & 0.03 \\
$L_{p m}$ & Length of PM & $\mathrm{mm}$ & 3 & 0.05 \\
$W_{s t}$ & tooth circumferential width & $\mathrm{mm}$ & 8 & 0.03 \\
$W_{p m}$ & PM circumferential width & $\mathrm{deg}$ & 12 & 0.05 \\
$H_{s y}$ & Height of stator yoke & $\mathrm{mm}$ & 31 & 0.06 \\
$H_{s p}$ & Height of stator plate & $\mathrm{mm}$ & 7 & 0.06 \\
$H_{s t}$ & Height of tooth & $\mathrm{mm}$ & 14.35 & 0.06 \\
$H_{p m}$ & Height of PM & $\mathrm{mm}$ & 15 & 0.05 \\
$N$ & Turns of coil & - & 75 & 0.5 \\
\hline \hline
\end{tabular}

The final optimal solution obtained from the proposed method is $\mathbf{x}=[10.5,11.55,6.6,3.1,0.85,3.15,8.25,11.71$, $32.64,15,6.35,12.45,90]$, according to the order $\mathbf{x}=\left[L_{s y}, L_{s p}\right.$, $\left.L_{\text {rot }}, L_{h d}, L_{g a p}, L_{p m}, W_{s t}, W_{p m}, H_{s y}, H_{s t}, H_{s p}, H_{p m}, N\right]$. The optimized tolerance is $\boldsymbol{\delta}_{\boldsymbol{x}}=[0.036,0.057,0.06,0.075,0.03,0.033,0.078$, $0.036,0.063,0.045,0.072,0.0330 .5]$. The optimized design has better performance, including the higher output power (599 $\mathrm{W}$ versus $500 \mathrm{~W})$ and efficiency $(83.1 \%$ versus $81.5 \%)$. Meanwhile, the temperature rise of in coil winding of the optimal solution is effectively limited under $75^{\circ} \mathrm{C}$. As illustrated in Fig. 12, the standard deviation of the output power is $1.12 \mathrm{~W}$ while the initial design is $1.69 \mathrm{~W}$. This means the less sensitive optimal solution is obtained with the optimized design parameters and their tolerances. As listed in Table V, the cost is adjusted, and the total cost is still lower than the initial one. Note that the manufacturing cost contains only the stator and PM manufacturing cost. The proposed method shows the effectiveness in conducting the robust motor optimization with constrained performance deviation while adjusting the cost.
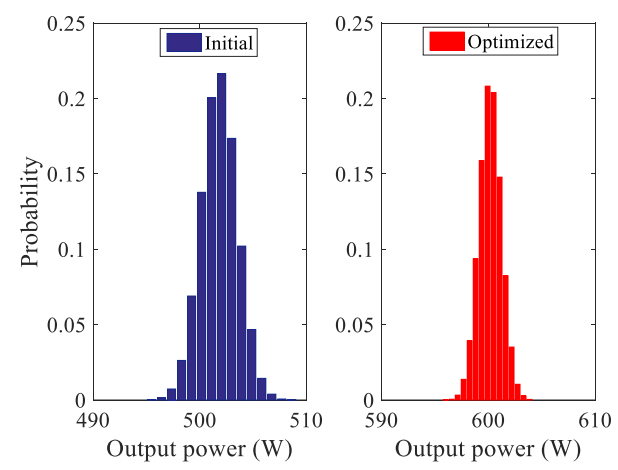

Fig. 12. Distribution of the output power

TABLE V

COST OF THE CPM

\begin{tabular}{llll}
\hline \hline Par. & Unit & Initial & optimal \\
\hline
\end{tabular}




\begin{tabular}{lccc} 
Material cost & AUD & 16.15 & 15.48 \\
Manufacturing cost & AUD & 2.85 & 3.28 \\
Total cost & AUD & 19 & 18.76 \\
\hline \hline
\end{tabular}

\section{CONCLUSION}

To take full advantage of SMC material for electrical machine developing, this paper present the relevant design, analysis and optimization approaches. The motor design experience and core loss modeling approach can benefit the SMC motor design and performance analysis. Considering the manufacturing, the orthogonal experiment design method can be used for analyzing and optimizing the SMC core properties effectively. For the tolerances in the actual production, the presented robust tolerance optimization based on DFSS can integrate the design optimization and production deeply with higher freedom considering performance, reliability, and cost.

\section{REFERENCES}

[1] Höganös AB, SOMALOY Technology for Electric Motors [Online].Available: www.hogonas.com.

[2] G. Lei, J. G. Zhu, and Y. G. Guo, Multidisciplinary Design Optimization Methods for Electrical Machines and Drive Systems, Springer, ISBN: 978-3-662-49269-7, 2016

[3] A. Krings, A. Boglietti, A. Cavagnino, and S. Sprague, "Soft magnetic material status and trends in electric machines," IEEE Trans. Ind. Electron, vol. 64, no. 3, pp. 2405-2414, 2017.

[4] B. Zhang, T. Seidler, R. Dierken, and M. Doppelbauer, "Development of a yokeless and segmented armature axial flux machine," IEEE Trans. Ind. Electron., vol. 63, no. 4, pp. 2062-2071, 2016.

[5] C. Liu, G. Lei, T. Wang, Y. Guo, Y. Wang, and J. Zhu, "Comparative study of small electrical machines with soft magnetic composite cores," IEEE Trans. Ind. Electron., vol. 64, no. 2, pp. 1049-1060, 2017.

[6] Y. Guo, J. G. Zhu, P. A. Watterson, and W. Wu, "Development of a PM transverse flux motor with soft magnetic composite core," IEEE Trans. Energy Convers., vol. 21, no. 2, pp. 426-434, 2006.

[7] Y. Li, J. Zhu, Q. Yang, Z. W. Lin, Y. Guo and C. Zhang, "Study on rotational hysteresis and core loss under three-dimensional magnetization, "IEEE Trans. Magn., vol. 47, no. 10, pp. 3520-3523, 2011.

[8] Y. Guo, J. Zhu, H. Lu, Y. Li, and J. Jin, "Core loss computation in a permanent magnet transverse flux motor with rotating fluxes," IEEE Trans. Magn., vol. 50, pp. 1-4, 2014.

[9] P. N. Koch, R. J. Yang, and L. Gu, "Design for six sigma through robust optimization," Struct. Multidiscip. Optim., vol. 26, no. 3/4, pp. 235-248, Feb. 2004.

[10] G. Lei, T. S. Wang, J. G. Zhu, et aI., "System level design optimization method for electrical drive system: robust approach," IEEE Trans. Ind. Electron., vol. 62, no. 8, pp.4702-47 I 3, Aug. 2015.

[11] B. Ma, G. Lei, J. Zhu, Y. Guo and C. Liu, "Application-oriented robust design optimization method for batch production of permanent magnet motors," IEEE Trans. Ind. Electron., to be published, doi: 10.1109/TIE.2017.2748046.

[12] B. Ma, G. Lei, C. Liu, J. Zhu and Y. Guo, "Robust tolerance design optimization of a PM claw pole motor with soft magnetic composite cores," IEEE Trans. Magn., to be published, doi: 10.1109/TMAG.2017.2756262. 